INPLASY

PROTOCOL

To cite: Chang Ma et al. Effect of black tea supplementation on blood pressure: a systematic review and doseresponse meta-analysis of randomized controlled trials. Inplasy protocol 202040021. doi:

10.37766/inplasy2020.4.0021

Received: 04 April 2021

Published: 04 April 2021

Corresponding author: peili bu

bupeili@medmail.com.cn

Author Affiliation:

Qilu Hospital of Shandong University

Support: no support

Review Stage at time of this submission: Piloting of the study selection process.

Conflicts of interest: None

\section{Effect of black tea supplementation on blood pressure: a systematic review and dose-response meta-analysis of randomized controlled trials}

\section{Chang Ma ${ }^{1}$.}

Review question / Objective: Does black tea supplementation have effect on blood pressure?.

Condition being studied: Cardiovascular disease (CVD) is the leading cause of morbidity, mortality, and disability worldwide. Hypertension is 1 of the leading causes of cardiovascular events and the main contributor to 7 million deaths annually worldwide. Even each difference of $20 \mathrm{mmHg}$ usual SBP is associated with more than a twofold difference in the stroke death rate and the death rates from IHD and from other vascular causes.

Information sources: Electronic searches. A comprehensive search for associated articles in electronic databases (pubmed, EMBASE, Cochrane Library) will be carried out from inception to the Match, 2020.0ther resources We will check and obtain potential studies from clinical trial registry, conference abstracts, and reference lists of relevant reviews.

INPLASY registration number: This protocol was registered with the International Platform of Registered Systematic Review and Meta-Analysis Protocols (INPLASY) on 04 April 2020 and was last updated on 04 April 2020 (registration number INPLASY202040021).

\section{INTRODUCTION}

Review question / Objective: Does black tea supplementation have effect on blood pressure?

Condition being studied: Cardiovascular disease (CVD) is the leading cause of morbidity, mortality, and disability worldwide. Hypertension is 1 of the leading causes of cardiovascular events and the main contributor to 7 million deaths annually worldwide. Even each difference of $20 \mathrm{mmHg}$ usual SBP is associated with more than a twofold difference in the stroke death rate and the death rates from IHD and from other vascular causes. 


\section{METHODS}

Search strategy: This systematic review and meta-analysis was conducted in accordance with the PRISMA statement guidelines. We searched PubMed, Embase, and the Cochrane Library from the index date of each database through March 2020. Titles and abstracts were searched for the following terms: Tea, black tea, flavonoid(s), tea extract(s), tea component(s), tea solid(s), camellia sinens, blood pressure, hypertension, hypertensive, hypotension, hypotensive , endothelium, endothelial function, and endothelial dysfunction. The search was limited to RCTs in adults (age > 18 years). Language was restricted to English.

Participant or population: Inclusion criteria :were conducted on human; Exclusion criteria: less than 18 years old.

Intervention: use of freshly brewed black tea or black tea powder produced by drying freshly brewed tea (not purified or isolated substances from tea).

Comparator: Placebo.

Study designs to be included: RCT.

Eligibility criteria: Were conducted on human; had a randomized controlled trial (RCT); use of freshly brewed black tea or black tea powder produced by drying freshly brewed tea (not purified or isolated substances from tea); assessed the effect of black tea on blood pressure; black tea was not administered as part of a multicomponent supplement in either the experimental or control group.

Information sources: Electronic searches. A comprehensive search for associated articles in electronic databases (pubmed, EMBASE, Cochrane Library) will be carried out from inception to the Match, 2020.0ther resources We will check and obtain potential studies from clinical trial registry, conference abstracts, and reference lists of relevant reviews.
Main outcome(s): Blood pressure between the intervention and control groups from baseline to the end of the intervention period.

Data management: Using Meta-Disc 1.4, Stata 15.0 and Review Manager 5.2 software for statistical analysis.

Quality assessment / Risk of bias analysis: Quality assessment / Risk of bias analysis: The risk of bias was applied according to the Cochrane guidelines including random sequence generation (selection bias), allocation concealment (selection bias), blinding of participants and personnel (performance bias), blinding of outcome assessment (detection bias), incomplete outcome data (attrition bias), selective reporting (reporting bias), and other sources. Only RCT will be assesed.

Strategy of data synthesis: Statistical analysis was performed with RevMan version 5 software (Cochrane Collaboration) and Stata V .16.0 software. Heterogeneity was assessed using the Cochran $Q$ statistic, in which a $P$ value of $<$ .10 was considered significant; and measured inconsistency (12) statistics, with 12> $50 \%$ considered indicative of substantial heterogeneity. $I^{2} \leq 50 \%$ implies homogeneity, and we will use a fixedeffects model. On the other hand, $I^{2}>50 \%$ reveals discrete heterogeneity, and we will exert a random-effects model.

Subgroup analysis: Whenever necessary, we will investigate sources of apparent heterogeneity based on the differences in consumption of black tea, duration of the black tea intervention; type of intervention; ethnicity; study design, et.

Sensibility analysis: Whenever necessary, we will examine the stability of study results by eliminating low quality studies.

\section{Countries involved: China}

Keywords: Blood pressure,black tea, metaanalysis. 